\title{
Krisenbewältigung geht vor, oder?
}

\section{Kinderrechte in der Pandemie}

Welche Rolle spielen die Rechte von Kindern und Jugendlichen in der Pandemie - ihr Recht auf Bildung, auf Gesundheit oder soziale Sicherheit? Wie sieht ihr Alltag aus, insbesondere der von Kindern, die in Armut leben? Wie wirken sich die Pandemiefolgen auf ihr Empfinden und Erleben aus? Kommen die notwendigen Hilfen an und wer ist besonders gefragt?

I n der Debatte um Kinderrechte spielen diese Aspekte eine große Rolle. Grundlegende Elemente des kindlichen Wohlergehens werden in der UN-Kinderrechtskonvention ausgehend von Artikel 3 in weiteren Artikeln konkretisiert. Sie zielen auf unterschiedliche Lebenslagedimensionen, u. a. auf Gesundheit (Artikel 24), soziale Sicherheit (Artikel 26), auf das Recht jedes Kindes auf den seiner körperlichen, geistigen, seelischen, sittli-

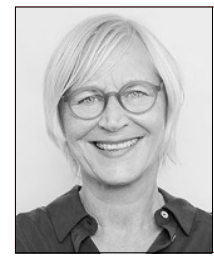

\section{Antje Richter-Kornweitz}

Hannover/Oldenburg, Deutschland

Dr., Dipl. Pädagogin, appr. Kinder- und Jugendlichenpsychotherapeutin. Landesvereinigung für Gesundheit und Akademie für Sozialmedizin Niedersachsen e. V.

antje.richter@gesundheit-nds.de

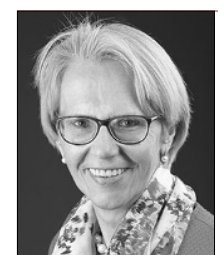

\section{Gerda Holz}

Frankfurt am Main, Deutschland

Sozialarbeiterin grad. und Diplom-Politikwissenschaftlerin; seit mehr als drei Jahrzehnten forschend und beratend zu (Kinder-) Armut und Armutsprävention tätig.

gerdaholz@t-online.de

Zusammenfassung Kinderrechte sind nicht verhandelbar, sondern von Gesellschaft und Staat jederzeit zu gewährleisten. Doch die COVID-19-Pandemie und deren bisherige Bewältigung haben junge Menschen weitreichend getroffen, ohne dass die Kinderrechte als Messlatte öffentlichen Handelns galten. Die öffentliche Diskussion um die Pandemiefolgen für Kinder und Jugendliche konzentrierte sich eher auf wenige Teilaspekte und auch die subjektive Perspektive junger Menschen auf das Geschehen wurde vernachlässigt. Im Beitrag wird dies skizziert und nicht nur ein Ausgleich pandemiebedingter Defizite gefordert, sondern eine an den Vorgaben der Kinderrechte ausgerichtete, klare Verbesserung ihrer Situation für ihr gelingendes Aufwachsen in Wohlergehen.

Schlüsselwörter Kinderrechte, Kinderarmut, Aufwachsen in Wohlergehen, Corona-Pandemie, Kommune chen und sozialen Entwicklung angemessenen Lebensstandard (Artikel 27), auf Bildung (Artikel 28) sowie auf Freizeit (Artikel 31). Hervorzuheben ist außerdem Artikel 19, der Schutz vor Gewaltanwendung thematisiert. Ergänzend wird dies unter Einbezug des familiären Kontexts betrachtet. Und auch wenn die in der UNKinderechtskonvention festgeschriebenen Kinderrechte mittlerweile den Status eines einfachen Bundesgesetzes haben, kommt deren Verwirklichung auf kommunaler Ebene bislang stark verzögert an (Bär et al. 2021).

Im Gegenteil, unter den Pandemie-Bedingungen der letzten beiden Jahre scheinen Kinderrechte kaum zu gelten, sie waren einfach „kein Thema“. Kinder und Jugendliche waren (und sind) quasi unsichtbar und zurückgeworfen auf den engsten Familienkreis. Sie tauchten erst langsam und ein knappes Jahr nach Pandemiebeginn als eigenständige Akteure und Betroffene im Gesellschaftsbild auf. Die Thematisierung ihrer Bedürfnisse angesichts der pandemiebedingten Einschränkungen beschränkte sich allerdings auf die - unbestreitbar bedeutenden - Fragen des Kinderschutzes und ihre allgemeine, überwiegend körperliche Unversehrtheit; letzteres jedoch meist nicht ohne auf die von ihnen ausgehenden Risiken als potenzielle Pandemietreiber aufmerksam zu machen.

\section{Diskussion um Pandemiefolgen für Kinder: auf wenige Teilaspekte reduziert}

Der Lebensalltag der jungen Menschen in den unterschiedlichen Lebensbereichen hat sich wie kaum ein anderer durch die Corona-Pandemie verändert. Dies wirkt sich auf ihr Empfinden und Erleben aus. Aber eine breite Diskussion über deren Folgen für die kindliche Entwicklung kommt erschreckend langsam und reduziert auf wenige Teilaspekte in Gang. Es geht dabei nicht um die Risiken zunehmender Entwicklungsrückstände für 


\section{Durchblick: Kinderrechte in Corona-Zeiten}

ein Aufwachsen in Wohlergehen und die Tatsache, dass Kindern die zeitnahe medizinische, therapeutische und/ oder pädagogische Förderung fehlt, die sie benötigen (Bt-Drs. 19/28274 2021). Genauswenig geht es darum, dass sich Früherkennungs- und Schuleingangsuntersuchungen verzögert haben, u. a. durch die völlige Überlastung des öffentlichen Gesundheitsdienstes, die zu Beginn geschlossenen Kitas und die im weiteren Pandemieverlauf häufig vorkommenden Schließungen einzelner Kitagruppen. Ebenso wenig steht im Fokus, dass die sonst ggf. durch Einrichtungen festgestellten Entwicklungsverzögerungen und Förderbedarfe der Kinder nicht erkannt werden und Vorbereitungen auf den Übergang „Familie/Kita“ oder „Kita/Grundschule“ oft komplett ausfallen. In diesem Kontext muss auch erwähnt werden, dass Ergebnisse von Schuleingangsuntersuchungen aus dem ersten Pandemiejahr zeigen, dass sich seitdem die schon zuvor bestehenden Entwicklungsunterschiede je nach sozio-ökonomischer Familienlage ausweiten, etwa im Bereich Motorik, beim Zählen oder bei der Sprachentwicklung (Bredahl 2021; Richter-Kornweitz und Holz 2021).

Viele junge Menschen sind viele Stunden allein, mit einem um bis zu 75 \% erhöhten Medienkonsum bei den 4- bis 17-Jährigen, ohne angerechnete Homeschooling-Zeiten. Im weiteren Pandemieverlauf ist damit zunehmender Bewegungsmangel verbunden, mit den bekannten negativen Folgen, wie eine Verschlechterung der Motorik oder Gewichtszunahme (Ärzteblatt.de 2020; Schmidt et al. 2021). Abgenommen haben bei 4bis 17-Jährigen vor allem Bewegung mit mittlerer und hoher Intensität, sportliche Aktivitäten in Verein und Freizeit, die zuvor für $70 \%$ der Kinder und Jugendlichen in Deutschland zum Alltag gehörten, sowie Alltagsaktivitäten wie Spazierengehen und (draußen) Spielen (Schmidt et al. 2021). Benachteiligt sind vor allem Kinder, die keinen Garten nutzen können und/oder in Mehrfamilienhäusern leben und allgemein Stadtkinder in Quartieren ohne ausreichenden Zugang zu Grünflächen. Dabei geht es um mehr als um einige Pfunde zu viel. Kinder entdecken die Welt in und durch Bewegung. Differenzierte Bewegungs- und Sinneserfahrungen, anregende Bewegungsmöglichkeiten und überhaupt das $\mathrm{Maß}$ an Bewegung sind entscheidende Promotoren in vielen Bereichen weit über die rein körperliche Gesundheit hinaus. Es geht um die Sprachentwicklung, die Entwicklung von Selbständigkeit und das Vertrauen in die eigenen Fähigkeiten, um soziales Lernen und viele weitere Entwicklungsbereiche.

Auch wenn es um Bildung geht, geht es kaum um ihr Recht darauf, sondern meist sogar um eine sehr begrenzte Bildungsvorstellung. Trotz der erheblichen An- strengungen der letzten Jahre um die Neuformatierung eines umfassenden Bildungsbegriffs („Bildung ist mehr als Schulbildung! “), in der Pandemie wird die Bedeutung von Bildung in Kindheit und Jugend nicht in voller Bandbreite diskutiert. Ausgeblendet bleiben häufig die anderen Lernwelten, die non-formalen und informellen Settings, in denen soziale, personale und instrumentelle Facetten der Bildung mit enormer Bedeutung für die Persönlichkeitsentwicklung vorwiegen. Das gesamte Spektrum der Bildungspotenziale jenseits von Schule hat Rauschenbach (2007) als ,andere Seite der Bildung“ oder „Alltagsbildung“ bezeichnet. Sieht man die Begrenzungen der Pandemie unter dieser Perspektive, dann bekommt das Ausmaß der Spielplatzsperrungen, der geschlossenen Jugendhäuser, Freizeittreffs und auch der Kitas, der Einschränkungen beim Sport oder auch beim Chillen/Abhängen mit Freund_innen eine ganz andere Bedeutung und dies erst recht, wenn man die ungleichen familiären Lebensbedingungen berücksichtigt. Diese Bildungsorte tragen außerhalb einer Pandemie dazu bei, Interessen zu wecken und zu fördern, Zugänge zu Kultur, zu Sport oder allgemein in die Gesellschaft zu eröffnen, kurz Teilhabe in Bereichen zu ermöglichen, die ansonsten verschlossen wären. In der Pandemie trifft dies junge Menschen in einer Altersphase, in der die Entwicklung normalerweise rasant vorangeht und ein Jahr weitaus mehr bedeutet als im Leben z. B. von Erwachsenen mittleren Alters. Damit fehlen die Entwicklungsimpulse, die gerade jetzt nötig wären.

Dominanter in der gesellschaftlichen und politischen Aufmerksamkeit ist dagegen die Institution „Schule“, vor allem ihre Organisation, die technische Ausstattung, die ständig wechselnden Formen des Unterrichtes, die Maßnahmen der Infektionsbekämpfung und des allgemeinen Infektionsschutzes. So spielen Abstands- und Hygienemaßgaben in Grundschulen seit Pandemiebeginn eine gewichtige Rolle. Dies alles führt national wie weltweit zu einer deutlichen Reduzierung der aktiven Lernzeit (Leopoldina 2021), die nicht ausgeglichen wird. Zwischen ein Viertel und die Hälfte aller Schüler_innen wandten in den Distanzphasen der Lockdowns weniger als zwei Stunden pro Tag für die Schule auf. Auch generell wird eine Verringerung der individuellen Lernzeit festgestellt, wobei die Arbeit der einzelnen Lehrkräfte und Schulen und deren Umgang mit der Pandemie eine entscheidende Rolle zu spielen scheint (Helm et al. 2021; Wößmann et al. 2021; Zinn und Bayer 2021; Statistisches Bundesamt 2022). Gleichzeitig öffnet sich die Schere in den digitalen Kompetenzen („Digital Divide“) zwischen verschiedenen sozioökonomischen Statusgruppen. Die Kompetenzen sind ungleich verteilt und stark vom Elternhaus beeinflusst, d. h. ab 
der 9. Klasse sind deutliche Unterschiede nach elterlicher Berufstätigkeit und Migrationshintergrund messbar (Bachmann et al. 2021).

Thematisiert wurden im weiteren Pandemieverlauf auch die Auswirkungen geschlossener Schulen und Kitas auf die Möglichkeiten von Eltern, die bislang üblichen Leistungen im Home-Office zu erbringen. Die Tatsache aber, dass Kinder viele Stunden des Tages allein verbringen müssen und somit Beschäftigungs- und Entwicklungsanreize ausbleiben, wenn die Eltern in sogenannten systemrelevanten, aber schlecht bezahlten Jobs arbeiten, wurde dabei meist ausgeblendet. Ebenso wenig stand die extreme Belastung der (meist weiblichen) Alleinerziehenden im Vordergrund, die häufig mit der Angst vor Jobverlust gepaart ist, wenn Betreuungsangebote ausfallen. Es ging eben nicht um die erheblichen Pandemiefolgen für den Bildungserfolg benachteiligter Gruppen, die im Endeffekt eine wachsende Bildungsungleichheit manifestieren, wie Studien aus europäischen Nachbarländern zeigen (Engzell et al. 2021).

\section{Einbußen bei Wohlbefinden und Lebensqualität \\ bei Kindern und Jugendlichen in Armutslagen}

Diese Entwicklung betrifft die jungen Menschen in allen Altersphasen und ganz besonders diejenigen in Armutslagen. So wird die Belastung für die Kinder in Familien mit niedrigem Haushaltseinkommen deutlich höher eingeschätzt als in jenen mit gesicherter Finanzlage. Psychische Auffälligkeiten berichten laut COPSY-Studie des Hamburger Universitätsklinikums Eppendorf (UKE) im ersten Jahr der Pandemie ein Drittel der 7- bis 17-Jährigen, während es vor der Krise lediglich zwei von zehn Kindern waren. Im zweiten Jahr haben Ängste und Sorgen bei den Kindern noch einmal deutlich zugenommen. Sie zeigen häufiger depressive Symptome und psychosomatische Beschwerden wie Bauch- und Kopfschmerzen oder Schlafprobleme (Ravens-Sieberer und Kaman 2021). Insgesamt weisen die Studien, die es bislang zu diesem Themenkomplex in Deutschland gibt, deutlich wachsende psychische Belastungen und Einbußen bezüglich des Wohlbefindens und der Lebensqualität bei 7- bis 17-Jährigen nach. Sie betreffen vor allem Familien mit jüngeren Kindern, mit niedrigem Sozialstatus, mit Migrationshintergrund und mit beengten Wohnverhältnissen (Schlack 2021).

Besonders jene Studien, die differenziertere Analysen nach Einkommen liefern, lassen allgemeine Schlussfolgerungen zu: Für armutsbetroffene junge Menschen war und ist ein Mithalten mit den Gleichaltrigen unmöglich. Sie leiden unter der Pandemie - vor allem durch fehlende Ausstattung zu Hause, durch begrenztere Erfahrungs- und Erlebnisräume, durch weniger Unterstüt- zung und Ersatz ausgefallener externer Ressourcen seitens der Eltern bzw. des sozialen Umfeldes, aber auch durch das zeitweilige Wegbrechen oder weiterhin andauernde Reduzierung der so wichtigen Kinder- und Jugendhilfeangebote (Geis-Thöne 2020; Andresen et al. 2021: MSGI 2021; Fendrich 2021). Damit sinken auch ihre Zukunftschancen massiv und es ist davon auszugehen, dass sich soziale Ungleichheit zu Lasten benachteiligter junger Menschen weiter vertieft. Hier haben die wenigen finanziellen Einmalzahlungen, die Sonderprogramme und die Verwaltungsvereinfachungen des Bundes, der Länder und Kommunen keine Trendwende bewirkt. Auch nicht das kreative und hohe individuelle Engagement vieler Fachkräfte vor Ort, was sehr wohl zu ganz neuen Arbeitsformen, aber eben auch zu hoher Arbeitsbelastung und zum Teil zur Selbsterschöpfung führte (Meyer 2021).

\section{Subjektive Perspektive von Kindern und Jugendli- chen kommt regelmäßig zu kurz}

Dieser einseitige Blick blieb aus „Kindperspektive“ nicht unbemerkt. Besonders kritisch bewerteten es beispielsweise Jugendliche, immer nur auf die Funktion als Schüler_innen beschränkt zu werden, anstatt mit allen ihren Bedürfnissen und ihrem Lebensalltag gesehen zu werden. Ebenso ist in der Pandemie die Unzufriedenheit unter Jugendlichen mit den Beteiligungsmöglichkeiten gerade in der Schule und darüber hinaus gestiegen: Nicht gehört zu werden wird als Ohnmachtsgefühl wahrgenommen (Andresen et al. 2021; Bertelsmann Stiftung/JuCo - Expert:innenteam Jugend und Corona 2021). Die Einbeziehung der subjektiven Perspektiven von Kindern und Jugendlichen auf das eigene Dasein, mit der sie als Akteur_innen mit ihrer eigenen Verortung in der Welt und ihren Anstrengungen zur Identitätsbildung in den Fokus rücken sollten, kommt regelmäßig zu kurz, nicht nur in der Pandemie. Anders gesagt, ihr Recht auf Gehör und Mitbestimmung (Artikel 12) wird verletzt.

\section{Fehlendes Zusammenspiel auf den politischen Ebenen verhindert Hilfen vor Ort}

Die öffentliche Verantwortung neben der elterlichen wurde bereits im 11. Kinder- und Jugendbericht (2002) definiert und ist als gemeinsame Aufgabe von Bund, Ländern und Kommunen zu verstehen. Entsprechend übernehmen alle Ebenen - allerdings meist mit höchst unterschiedlichen Zielvorstellungen, Themensetzungen und Maßnahmenplänen - wichtige Aufgaben - gerade jetzt.

Während Bund und Länder vorrangig finanzielle und gesetzliche Regelungen sowie unterstützende Förder- 


\section{Durchblick: Kinderrechte in Corona-Zeiten}

programme zu verantworten haben, ist es Aufgabe der Kommunen durch Vorhaltung lokaler Infrastruktur und Gestaltung des gemeinschaftlichen Lebens ein gelingendes Aufwachsen in Wohlergehen zu fördern, umfassende Teilhabechancen zu eröffnen und dazu die Perspektive von Kindern und Jugendlichen auf Bedarf und Bedürfnisse zu beachten. In der Pandemie sind die Schwachstellen jeder Ebene sowie ihres Zusammenwirkens mehr als deutlich geworden (Klundt und Müller 2020; Holz und Kornweitz 2020). Anzahl und Ausrichtung der Aktivitäten sind mittlerweile zwar beachtlich, was aber nichts über deren Bedarfsgerechtigkeit, Passgenauigkeit und zielgerichtete Wirkungen aussagt. Die von den Fachministerien aufgelegten Bundesprogramme wie „Digitalpakt Schule“ (https://www.digitalpaktschule.de/) oder das Aktionsprogramm „Aufholen nach Corona für Kinder und Jugendliche" (https://www. bmfsfj.de/bmfsfj/themen/corona-pandemie/aufholennach-corona) gehen auf in Landesprogrammen mit jeweils eigenen Akzenten, eigenen Verwaltungsregularien, auf jeweils eigenen Onlineportalen. Dies führt zu verlangsamter Umsetzung vor Ort, zu neuer Intransparenz und Unübersichtlichkeit, zu einem immensen Rechercheaufwand für Einzelpersonen, Fachkräfte und Institutionen. Eine sichtbare Folge ist, dass endlich bereitgestellte Bundes-/Landesgelder nicht beantragt werden, weil Personal fehlt oder mit anderen Formen der Krisenbewältigung ausgefüllt ist (Frenzel 2021).

Die berechtigten und wiederkehrenden Forderungen aus Wissenschaft, Praxis und Interessenverbänden an Bund, Länder und Kommunen, Wohlbefinden und Rechte der Kinder zum Maßstab der politischen Entscheidungen zu machen, werden so konterkariert, statt gemeinsam vor Ort entsprechende Bedingungen des Aufwachsens zu schaffen.

\section{Die Kommune als Garant gelebter Kinderrechte - jetzt und morgen}

Aufgabe von Kommunen ist, gelingendes Aufwachsen in Wohlergehen zu fördern und fehlende Teilhabechancen von Kindern und Jugendlichen aktiv auszugleichen. Dabei werden sie - wie zuvor skizziert - mit der ungleichen Verteilung von Teilhabe- und Entwicklungschancen konfrontiert, mit ungleichen Chancen auf Gesundheit, Bildung und soziale Teilhabe, welche in engem, wechselseitigem Zusammenhang zur materiellen Lage der Familie stehen. Kommunen müssen sich ebenfalls mit dem Phänomen auseinandersetzen, dass Menschen mit besonders hohem Belastungsgrad durch präventive Angebote häufig weniger gut erreicht werden als andere („Präventionsdilemma“; vgl. Bauer 2005). Vor der Herausforderung, den Folgen dieser Ungleichheit - auch je- ner, die durch Entwicklungen jenseits der direkten kommunalen Einflussmöglichkeiten bedingt sind - aktiv zu begegnen und umfassende Teilhabe bedarfs- und bedürfnisgerecht zu realisieren, stehen alle Kommunen in Deutschland. Jedoch nicht alle greifen die Aufgaben im Rahmen ihrer Möglichkeiten und Ausgangsbedingungen aktiv und umfassend genug auf. Tatsächlich zeigen sich sehr diverse kommunale Landschaften, regionale Ungleichheiten und Trends zunehmender räumlicher, regionaler und quartiersbezogener Ungleichheit, die den Lebensalltag von Kindern und Jugendlichen prägen und dem Ziel entgegenstehen, ihnen bestmögliche Bedingungen des Aufwachsens zu gewährleisten.

In der Pandemie wurden und werden die Schwachstellen im kommunalen Organisationsgefüge besonders deutlich. Personalmangel und Arbeitsüberlastung, fehlende technische Ausstattung, fehlende Qualifikation, schwergängige Routinen und Probleme, schnell und flexibel reagieren zu können sowie das immer noch weit verbreitete Denken in Zuständigkeiten kommen nicht in jeder Kommune, aber immer noch zu häufig, vor. Im Gegensatz zu den Pflichtaufgaben steckt die Entwicklung nachhaltiger präventiver Strukturen vor Ort noch in den Kinderschuhen, abgesehen von Leuchtturmprojekten und einzelnen Ansätzen auf Bundeslandebene.

Das Aufwachsen im Wohlergehen für alle zu sichern, ist dagegen aktuell und künftig ein bedeutendes kinder- und jugendpolitisches Ziel, das soziale, entwicklungspsychologische und politische sowie planerische Elemente vereint und das Zusammenwirken der verschiedenen politischen Ebenen erfordert (Kruse und Richter-Kornweitz 2022). Es beinhaltet die aktive, strategisch ausgerichtete Gestaltung der Rahmenbedingungen über die Bereitstellung multipler Ressourcen und die Förderung integrativer Prozesse. Dabei handelt sich um einen mittel- bis langfristigen, strategiegeleiteten Prozess, der zu einer nachhaltig verankerten (Infra-)Struktur führt, als entscheidender Beitrag zur Vermeidung von Armutsfolgen bei jungen Menschen. Ausgangspunkt für eine verlässliche kommunale Präventionsstruktur in diesem Sinne ist die Überzeugung, dass präventive Leistungen für Kinder und ihre Familien kommunale Pflichtaufgabe sind und ausgehend von Bedarf und Bedürfnissen bereitgestellt werden - nicht aus der Handlungslogik einzelner Ressorts. Eine solche Veränderung des kommunalen Systems erfordert einen Paradigmenwechsel und eine programmatisch ausgerichtete Politik, die die Belange armer Kinder oben auf die Agenda setzt - und damit aktive Prävention verfolgt, anstatt auf Defizite zu reagieren. Zuvorderst muss dazu der kommunale Wille vor Ort da sein und politisch ab- 
gesichert werden. Politik und Verwaltung dürfen sich selbst nicht aus der Pflicht nehmen.

\section{Statt eines Fazits - Eine Vorgabe für die Zukunft}

Kinderrechte sind nicht verhandelbar, sondern von Gesellschaft und Staat jederzeit zu gewährleisten. Die Pandemie und deren bisherige Bewältigung zeigen, wie weitreichend junge Menschen von den Auswirkungen betroffen sind und wie wenig dies zur zentralen Messlatte öffentlichen Handelns wird. Vorgabe für die $\mathrm{Zu}$ kunft ist, schnellstens eine umfassende Neuausrichtung vorzunehmen, bei der es nicht allein um den Ausgleich pandemiebedingter Defizite geht, sondern um eine an den Vorgaben der Kinderrechte ausgerichtete, klare Verbesserung ihrer Situation .

Eingegangen. 31. Januar 2022

Angenommen. 4. Februar 2022

\section{Literatur}

Andresen, S., Heyer, L., Lips, A., Rusack, T., Schröer, W., Severine, T., \& Wilmes, J. (2021). Das Leben von jungen Menschen in der Corona-Pandemie. Erfahrungen, Sorgen, Bedarfe. Gütersloh: Bertelsmann Stiftung.

Ärzteblatt.de (Hrsg.). (2020). Kinder bewegen sich weniger, essen schlechter und konsumieren mehr Medien. https://www.aerzteblatt.de/nachrichten/119111/Kinder-bewegen-sich-weniger-essen-schlechter-und-konsumieren-mehr-Medien. Zugegriffen: 17. Febr. 2022

Bachmann, R., Hertweck, F., Kamb, R., Lehner, J., Niederstadt, M., \& Rulff, C. (2021). Diskussionspapier. Digitale Kompetenzen in Deutschland - eine Bestandsaufnahme. RWI Materialien Heft 15. Essen: RWI - LeibnizInstitut für Wirtschaftsforschung.

Bär, D., Roth, R., \& Csaki, F. (Hrsg.). (2021). Handbuch kinderfreundliche Kommunen. Kinderrechte kommunal verwirklichen. Frankfurt am Main: Debus Pädagogik Verlag.

Bauer, U. (2005). Das Präventionsdilemma. Potenziale schulischer Kompetenzförderung im Spiegel sozialer Polarisierung. Wiesbaden: VS Verlag für Sozialwissenschaften

Bertelsmann (Hrsg.). (2021). JuCo-Expert:innenteam Jugend und Corona. „Fragt uns 2.0" - Corona-Edition. Anmerkungen von jugendlichen Expert:innen zum Leben von Kindern und Jugendlichen in der Pandemie. Gütersloh. https://www.bertelsmann-stiftung.de/de/publikationen/publikation/did/fragt-uns-20. Zugegriffen: 31. Jan. 2022.

BMFSFJ - Bundesministerium für Familie, Senioren, Frauen und Jugend (Hrsg.). (2002). Elfter Kinder- und Jugendbericht. Bericht über die Lebenssituation junger Menschen und die Leistungen der Kinder- und Jugendhilfe in Deutschland. Berlin.

Bredahl, R. (2021). Kleine Kinder, kleine Sorgen? Folgen der Pandemie für Vorschulkinder. Folienvortrag auf dem 2. Düsseldorfer Symposium zu Kinderrechten und Kinderschutz - Kinder und Jugendliche in der Pandemie, 24. März 2021.

BT-Drs. 19/28274 = Bundestagsdrucksache 19/28274 (2021). Bildungsund Gesundheitschancen von Kindern und Jugendlichen während der Corona-Pandemie. Antwort der Bundesregierung auf die Kleine Anfrage der Abgeordneten Pascal Kober, Michael Theurer, Johannes Vogel (Olpe), weiterer Abgeordneter und der Fraktion der FDP - Drucksache 19/27708. https://dip21.bundestag.de/dip21/btd/19/282/1928274.pdf. (Letzter Onlinezugriff: 31. Jan. 2022)

Engzell, P., Frey, A., \& Verhagen, M. (2021). Learning loss due to school closures during the COVID-19 pandemic, SocArXiv papers. https://osf.io/ preprints/socarxiv/ve4z7/. Zugegriffen: 31. Jan. 2022.
Fendrich, S. (2021). Weniger Erziehungshilfen - Eine Folge von Corona. In: KomDat 3/2021 (S. 5-7). Dortmund: Dortmunder Arbeitsstelle Kinder\& Jugendhilfestatistik. https://www.akjstat.tu-dortmund.de/fileadmin/68_ KomDat_3_21.pdf. Zugegriffen: 17. Febr. 2022

Frenzel Chr. (2021). Nachgefragt: Mehr Gesundheitsförderung und Prävention für Kinder und Jugendliche - ein Baustein zur Überwindung der Pandemie? Mainz. https://www.jugendhilfeportal.de/fokus/gesundheit/arti$\mathrm{kel} /$ interview-zum-ima-bericht-erste-schritte-aber-kein-paradigmenwechsel/. Zugegriffen: 17. Febr. 2022.

Geis-Thöne, Wido (2020). Häusliches Umfeld in der Krise: Ein Teil der Kinder braucht mehr Unterstützung - Ergebnisse einer Auswertung des Sozio-oekonomischen Panels (SOEP). Köln: Institut der deutschen Wirtschaft https://www.iwkoeln.de/fileadmin/user_upload/Studien/Report/PDF/2020/ IW-Report_2020_Haeusliche_Lebenswelten_Kinder.pdf. Zugegriffen: 17. Feb. 2022.

Helm, C., Huber, S.G., Postlbauer, A. (2021). Lerneinbußen und Bildungsbenachteiligung durch Schulschließungen während der Covid-19-Pandemie im Frühjahr 2020. Eine Übersicht zur aktuellen Befundlage, in: Fickermann, D., Edelstein, B., Gerick, J., Racherbäumer, K. (Hrsg.). Schule und Schulpolitik während der Corona-Pandemie. Nichts gelernt?, S. 59-81. Münster; New York: Waxmann 2021.

Holz, G., \& Richter-Kornweitz, A. (2020). Corona-Chronik. Gruppenbild ohne (arme) Kinder. Eine Streitschrift. ISS: Frankfurt a.M.; LVG \& AFS: Hannover. https://www.praeventionsketten-nds.de/fileadmin/media/downloads/Coronachronik_Streitschrift_final.pdf. Zugegriffen: 17. Febr. 2022.

Klundt, M., \& Müller, N. (2020). Krisengerechte Kinder statt kindergerechtem Krisenmanagement? Auswirkungen der Corona-Krise auf die Lebensbedingungen junger Menschen. Berlin: DIE LINKE. im Bundestag.

Kruse, C., \& Richter-Kornweitz, A. (2022). Positionen. Präventionsketten verankern. Landesvereinigung für Gesundheit und Akademie für Sozialmedizin Niedersachsen e. V., Hannover. https://www.praeventionsketten-nds. de/fileadmin/media/downloads/Positionspapier/Positionspapier_Praeventionsketten-verankern.pdf. Zugegriffen: 31. Jan. 2022.

Leopoldina - Nationale Akademie der Wissenschaften (2021). Kinder und Jugendliche in der Coronavirus-Pandemie: psychosoziale und edukative Herausforderungen und Chancen. 8. Ad-hoc-Stellungnahme vom 21. Juni 2021, Halle/Saale. https://www.leopoldina.org/uploads/tx_leopublication/2021_Corona_Kinder_und_Jugendliche.pdf. Zugegriffen: 31. Jan. 2022.

Meyer, U. (2021). Fact sheet: Studie „Soziale Arbeit Macht Gesellschaft“, Fulda. https://www.verdi.de/++file++604f7ced38173fdb3859f46b/download/Fact \%20sheet_Soziale\%20Arbeit \%20macht\%20Gesellschaft.pdf. Zugegriffen: 31. Jan. 2022.

MSGI = Ministerium für Soziales, Gesundheit und Integration BadenWürttemberg-Schwerpunkt Strategie (2021). „Starke Kinder - chancenreich“-Arbeitspapier „Zusammenstellung der Rückmeldungen der Abfrage bei Projekten gegen Kinderarmut in Baden-Württemberg “, 12/2021. Stuttgart: MSGI.

Rauschenbach, T. (2007). Im Schatten der formalen Bildung. Alltagsbildung als Schlüsselfrage der Zukunft. In: Diskurs Kindheits- und Jugendforschung 2 (2007) 4, S. 439-453. https://www.pedocs.de/volltexte/2009/1024/pdf/Rauschenbach_Im_Schatten_Diskurs_2007_4_D.pdf. Zugegriffen: 31. Jan. 2022.

Ravens-Sieberer, U., \& Kaman, A. (2021). Seelische Gesundheit und psychische Belastungen von Kindern und Jugendlichen in der ersten Welle der COVID-19-Pandemie - Ergebnisse der COPSY-Studie. Bundesgesundheitsblatt - Gesundheitsforschung - Gesundheitsschutz. https://doi.org/10.1007/s00103-021-03291-3.

Richter-Kornweitz, A., \& Holz, G. (2021). Corona-Chronik - Gruppenbild ohne (arme) Kinder. Eine Streitschrift. In Deutsche Gesellschaft für Sozialpädiatrie und Jugendmedizin (DGSPJ) (Hrsg.), Kinderärztliche Praxis. Soziale Pädiatrie und Jugendmedizin und auf "Sozialpädiatrie aktuell“ (S. 110-111). Heft 4/2021.

Schlack, R. (2021). Schlaglicht: Psychische Gesundheit von Kindern und Jugendlichen in der COVID-19-Pandemie. https://www.apk-ev.de/fileadmin/ downloads/Materialien_KiJu/Praesentationen_Abschlussworkshop/APKFachtag01.06.2021_RKI_Schlaglicht_COVID-19_Kinder_final_publ.pdf. Zugegriffen: 31. Jan. 2022. 
Sozial Extra 22022

Durchblick: Kinderrechte in Corona-Zeiten

Schmidt, S. C. E., Burchartz, A., Kolb, S., Niessner, C., Oriwol, D., Hanssen-Doose, A., Worth, A., \& Woll, A. (2021). Zur Situation der körper-

lich-sportlichen Aktivität von Kindern und Jugendlichen während der CO-

VID-19 Pandemie in Deutschland. Die Motorik-Modul Studie (MoMo).

https://www.sport.kit.edu/rd_download/1.\%20Startseite/Aktuelles/

Schmidt\%20et\%20al\%20MoMo\%20Corona\%202\%20SR\%202021.pdf. Zugegriffen: 31. Jan. 2022.

Statistisches Bundesamt (2022). „93.100 Schülerinnen und Schüler wiederholten im Corona-Schuljahr 2020/2021 die Klassenstufe“. Pressemitteilung Nr. N 002 vom 21. Januar 2022, Wiesbaden. https://www.destatis.de/ DE/Presse/Pressemitteilungen/2022/01/PD22_N002_21.html;isessionid=9 BD5CA7B21B7435E017DA082FFFE1B12.live712. Zugegriffen: 01. Mär. 2022.

Wößmann, L., Freundl, V., Grewenig, E., Lergetporer, P., Werner, K., \& Zierow, L. (2021). „Bildung erneut im Lockdown: Wie verbrachten Schulkinder die Schulschließungen Anfang 2021? “ ifo Schnelldienst 74 (05),

München. https://www.ifo.de/publikationen/2021/aufsatz-zeitschrift/bildung-erneut-im-lockdown-wie-verbrachten-schulkinder-die. Zugegriffen: 31. Jan. 2022.

Zinn, S., \& Bayer, M. (2021). „DIW Berlin: Schule in der Pandemie: Lernzeiten der Kinder hängen auch von der Bildung der Eltern ab“. DIW aktuell 63, Berlin. https://www.diw.de/documents/publikationen/73/ diw_01.c.817844.de/diw_aktuell_63.pdf. Zugegriffen: 31. Jan. 2022. 